\title{
ON THE OSCILLATION FUNCTIONS OF GAUSSIAN PROCESSES
}

\author{
KIYOSI ITÔ and MAKIKO NISIO
}

\section{Introduction and results obtained.}

By a Gaussian process we shall understand a separable, measurable, jointly Gauss distributed process with the time parameter on $[0,1]$, continuous in the (second order) mean.

As to the regularity of the sample path of a stationary Gaussian process, we have $\mathrm{Yu}$. K. Belayev's theorem of alternatives [1] which reads as follows: the sample function (path) of a stationary Gaussian process is either continuous with probability one or unbounded on every interval with probability one.

What will happen for a non-stationary Gaussian process? Our purpose is to answer this question.

Given a Gaussian process $x=x(t, \omega), 0 \leqq t \leqq 1, \omega \in \Omega(\mathscr{B}, P)$, we shall define the oscillation function of $x$ by

$$
W_{x}(t, \omega)=\lim _{\varepsilon \downarrow 0} \sup _{u, v \in(t-\varepsilon, t+\varepsilon) \cap[0,1]}|x(v, \omega)-x(u, \omega)|,
$$

where we apply the usual convention $(+\infty)-(+\infty)=(-\infty)-(-\infty)=0$, etc. Because of the separability of our process $x$, the supremum can be taken only for the $u$ and $v$ in the separant $Q$ of $x$ which is countable, so that $W_{x}(t, \omega)$ is measurable in $\omega$ for each $t \in[0,1]$.

An interesting fact is that the oscillation function of a Gaussian process is a deterministic function. Precisely speaking, we have the following theorem which will be proved in Section 2 .

Theorem 1. There exists a function $\alpha=\alpha_{x}(t), 0 \leqq t \leqq 1$, which does not depend on $\omega$, such that

$$
P\left[W_{x}(t, \omega)=\alpha(t) \text { for every } t \in[0,1]\right]=1 .
$$

In view of this theorem we call $\alpha$ the oscillation function of the Gaussian process $x$.

Received August 25, 1967.

Math. Scand. $22-14$ 
Using the fact that the probability law of the process $x(t)-E(x(t))$, $0 \leqq t \leqq 1$, is invariant under reflection, we shall prove the following theorem in Section 3 :

Theorem 2. For each $t \in[0,1]$, we have

$$
P\left[\varlimsup_{s \rightarrow t} x(s)=x(t)+\frac{1}{2} \alpha(t), \varliminf_{s \rightarrow t} x(s)=x(t)-\frac{1}{2} \alpha(t)\right]=1 .
$$

It is clear that, with probability one, the sample function of $x(t)$ is continuous at every point $t$ where $\alpha(t)$ vanishes.

We have also the following theorem which will be proved in Section 4 .

Theorem 3. If, for some constant $a, \alpha(t) \geqq a>0$ on a dense subset $D$ of an open interval $I \subset[0,1]$, then

$$
P\left[\varlimsup_{s \rightarrow t} x(s)=\infty, \varliminf_{s \rightarrow t} x(s)=-\infty \text { for every } t \in I\right]=1 .
$$

In Section 5 and 6 we shall prove the following properties that characterize oscillation functions.

THeOREM 4. (a) The osoillation function of a Gaussian process satisfies $\alpha(t)$ is upper semi-continuous,

$$
\{t: a \leqq \alpha(t)<\infty\} \text { is nowhere dense for every } a>0 .
$$

(b) Conversely, given a function $\alpha:[0,1] \rightarrow[0, \infty]$ satisfying $(\alpha, 1)$ and $(\alpha, 2)$, we can construct a (not necessarily unique) Gaussian process whose oscillation function is $\alpha$.

Let us derive Belayev's theorem of alternatives from Theorems 1 and 3. Suppose $x(t)$ is a stationary Gaussian process. The oscillation function $\alpha(t)$ of the restriction of $x$ to $0 \leqq t \leqq 1$ is constant because of the stationarity. If the constant is 0 , then almost all sample functions of $x$ are continuous; if it is positive, then almost all sample functions are, by Theorem 3, unbounded both below and above on every interval.

\section{Proof of Theorem 1.}

We can assume that $E x(t)=0$, because $E x(t)$ is continuous in $t$. Let $R(s, t), t, s \in[0,1]$, be the covariance function of $x$. Then $R$ is real, symmetric, positive-definite and continuous on $I \times I$. Using Mercer's theorem, we can expand $R$ as follows: 


$$
R(t, s)=\sum_{n} \varphi_{n}(t) \varphi_{n}(s) / \lambda_{n},
$$

where $\left\{\lambda_{n}\right\}$ and $\left\{\varphi_{n}\right\}$ are, respectively, the positive eigenvalues and the corresponding real (normalized) eigenfunctions for the integral operator with the kernel $R(t, s)$, that is,

$$
\varphi_{n}(t)=\lambda_{n} \int_{0}^{1} R(t, s) \varphi_{n}(s) d s, \quad 0 \leqq \lambda_{1} \leqq \lambda_{2} \leqq \ldots,
$$

and the sum in (2.1) converges absolutely and uniformly on $I \times I$.

We shall define a sequence of random variables $x_{n}, n=1,2, \ldots$, as the Fourier coefficients of the sample function of $x(t)$ with respect to $\left\{\varphi_{n}\right\}$ :

$$
x_{n}=\int_{0}^{1} x(t) \varphi_{n}(t) d t .
$$

Observing that

we have

$$
E\left[\int_{0}^{1} x(s)^{2} d s\right]=\int_{0}^{1} R(s, s) d s<\infty,
$$

$$
P\left[\int_{0}^{1} x(s)^{2} d s<\infty\right]=1,
$$

so that $x_{n}$ is well-defined.

A simple computation shows that

$$
E\left(x_{n} x_{m}\right)=0, n \neq m, \quad E\left(x_{n}{ }^{2}\right)=\lambda_{n}^{-1},
$$

which implies that $x_{n}, n=1,2, \ldots$, are independent, each having the Gauss distribution with mean 0 and variance $\lambda_{n}{ }^{-1}$. Observing that

$$
E\left[\left|x(t)-\sum_{1}^{N} \varphi_{n}(t) x_{n}\right|^{2}\right]=R(t, t)-\sum_{1}^{N} \lambda_{n}{ }^{-1} \varphi_{n}(t)^{2} \rightarrow 0
$$

as $n \rightarrow \infty$, we have

$$
P\left[x(t)=\sum_{n=1}^{\infty} \varphi_{n}(t) x_{n}\right]=1 \quad \text { for each } t
$$

the infinite series converges to $x(t)$ in the mean for each $t$ and so it converges with probability one for each $t$ because of the independence of $x_{n}$, $n=1,2, \ldots$.

Let us define the maximum oscillation $W_{y}(s, t, \omega)$ of a separable process on the interval $[s, t]$ by 


$$
W_{y}(s, t, \omega)=\lim _{n \uparrow \infty} \lim _{p \uparrow \infty} \sup _{\substack{u, v \in\left(s-n^{-1}, t+n^{-1}\right) \cap[0,1] \\|u-v|<p^{-1}}}|y(u, \omega)-y(v, \omega)|,
$$

where the interval $[0,1]$ can be replaced by the separant $Q$ of $y$.

We shall now prove that $W_{x}(s, t, \omega)$ is a constant; more precisely, that there exists a function $\alpha(s, t)$ independent of $\omega$ such that

$$
P\left[W_{x}(s, t, \omega)=\alpha(s, t)\right]=1
$$

for each pair $s \leqq t$.

Since $\varphi_{j}(t)$ is continuous in $t$, we have

where

$$
P\left[W_{x}(s, t, \omega)=W_{y_{n}}(s, t, \omega)\right]=1,
$$

$$
y_{n}(t)=x(t)-\sum_{j=1}^{n} \varphi_{j}(t) x_{j}=\sum_{j=n+1}^{\infty} \varphi_{j}(t) x_{j} .
$$

The separant $Q$ of $x$ is also that of $y_{n}$ because of the continuity of $\varphi_{j}(t)$. By virtue of (2.4), $y_{n}(t)$ is measurable with respect to $\mathscr{B}\left(x_{k}, k \geqq n\right)$ for each $t$. But $W_{y_{n}}(s, t, \omega)$ is measurable with respect to $\mathscr{B}\left(y_{n}(t), t \in Q\right)$. Since $Q$ is countable, $W_{y_{n}}(s, t, \omega)$ is measurable with respect to $\mathscr{B}\left(x_{k}, k \geqq n\right)$ and so $W_{x}(s, t, \omega)$ is also measurable with respect to $\mathscr{B}\left(x_{k}, k \geqq n\right)$ for every $n$, by (2.7). Since the $x_{n}, n=1,2, \ldots$, are independent, Kolmogorov's zero-one law shows that $W_{x}(s, t, \omega)$ is a constant with probability one.

We shall now strengthen (2.6) to get

$$
P\left[W_{x}(s, t, \omega)=\alpha(s, t) \text { for every pair } s \leqq t\right]=1 .
$$

It follows from (2.6) that

$\left(2.6^{\prime \prime}\right) \quad P\left[W_{x}(s, t, \omega)=\alpha(s, t)\right.$ for every rational pair $\left.s \leqq t\right]=1$.

Since $W_{x}(s, t, \omega)$ is left-continuous in $s$ and right-continuous in $t,\left(2.6^{\prime \prime}\right)$ implies $\left(2.6^{\prime}\right)$. Writing $W_{x}(t, \omega)$ and $\alpha(t)$ for $W_{x}(t, t, \omega)$ and $\alpha(t, t)$, respectively, we get from $\left(2.6^{\prime}\right)$

$$
P\left[W_{x}(t, \omega)=\alpha(t) \text { for every } t\right]=1 .
$$

\section{Proof of Theorem 2.}

We shall use the same notation as in Section 2. Using Kolmogorov's zero-one law in the same way as before, we can see that $\varlimsup_{s \rightarrow t}(x(s, \omega)-$ $x(t, \omega))$ is a constant, say $\beta(t)$, with probability one. Since the process $y(t) \equiv-x(t)$ has the same Gaussian probability law as the process $x$, we have

$$
\varlimsup_{s \rightarrow t}(-x(s)+x(t))=\beta(t),
$$


that is,

$$
\lim _{s \rightarrow t}(x(s)-x(t))=-\beta(t)
$$

with probability one. By definition we have

$$
\begin{aligned}
W_{x}(t, \omega) & =\varlimsup_{s \rightarrow t} x(s)-\varliminf_{s \rightarrow t} x(s) \\
& =\varlimsup_{s \rightarrow t}(x(s)-x(t))-\varliminf_{s \rightarrow t}(x(s)-x(t)) .
\end{aligned}
$$

Therefore, $\alpha(t)$ must be equal to $2 \beta(t)$. This completes the proof of Theorem 2.

\section{Proof of Theorem 3.}

In the following lemma and throughout this paper an open subset of $[0,1]$ means a subset open in $[0,1]$. For example, $[0, u)$ is open and 0 is an interior point of this interval.

Lemma 4.1. Let $x(t), 0 \leqq t \leqq 1$, be a separable process continuous in probability, and $D$ a dense subset of an open subinterval $I$ of $[0,1]$. For each $t \in I$, we can then find a sequence $s_{n} \in D$ such that $s_{n} \rightarrow t$ and that

$$
P\left[\varlimsup_{n \rightarrow \infty} x\left(s_{n}\right)=\varlimsup_{s \rightarrow t} x(s)\right]=1
$$

and hence a fortiori

$$
P\left[\varlimsup_{\substack{s \rightarrow t \\ s \in D}} x(s)=\varlimsup_{s \rightarrow t} x(s)\right]=1
$$

Proof. Let $Q=\left\{t_{n}\right\}$ be a separant of $x(t)$ and let $\left\{U_{n}\right\}$ be a sequence of neighborhoods of $t$ converging to $t$. Let $\left\{y_{n}(\omega)\right\}$ be a sequence converging to $\bar{x}(t)=\varlimsup_{s \rightarrow t} x(t)$ strictly from below, for example

$$
y_{n}(\omega)=\min (\bar{x}(t), n)-1 / n \text {. }
$$

Since $Q$ is a separant of $x(t)$, we can find $u_{n 1}, u_{n 2}, \ldots, u_{n p_{n}} \in U_{n} \cap Q$ such that

$$
P\left[\max _{k} x\left(u_{n k}\right)>y_{n}(\omega)\right]>1-2^{-n} .
$$

By Borel-Cantelli's lemma we have

$$
P\left[\lim _{n} \max _{k} x\left(u_{n k}\right) \geqq \bar{x}(t)\right]=1 .
$$

Writing $\left\{u_{n}\right\}$ for $\left\{u_{11}, \ldots, u_{1 p_{1}}, u_{21}, \ldots, u_{2 p_{2}}, \ldots\right\}$, we have 


$$
P\left[\varlimsup_{n \rightarrow \infty} x\left(u_{n}\right) \geqq \bar{x}(t)\right]=1 .
$$

Since $x(t)$ is continuous in probability, we can find

such that

$$
s_{n} \in\left(u_{n}-1 / n, u_{n}+1 / n\right) \cap D
$$

$$
P\left[\left|x\left(u_{n}\right)-x\left(s_{n}\right)\right|>2^{-n}\right]<2^{-n}, \quad n=1,2, \ldots .
$$

Using Borel-Cantelli's lemma again, we have

$$
P\left[\overline{\lim }_{n} x\left(u_{n}\right)=\varlimsup_{n} x\left(s_{n}\right)\right]=1,
$$

which, combined with (4.2), implies (4.1) and so (4.1').

We shall now prove Theorem 3. Using (4.1) and Theorem 2, we can see that the event

$$
\Omega_{1}=\left\{\omega: \varlimsup_{\substack{s \rightarrow t \\ s \in D}} x(s, \omega)=x(t, \omega)+\frac{1}{2} \alpha(t) \text { for every } t \in D\right\}
$$

has probability one, since $D$ is a countable dense subset of $I$. For every $\omega \in \Omega_{1}$ and every $t \in D$, we have

$$
\begin{aligned}
\varlimsup_{\substack{s \rightarrow t \\
s \in D}} x(s, \omega) & =\varlimsup_{\substack{s \rightarrow t \\
s \in D}} \varlimsup_{\substack{u \rightarrow s \\
u \in D}} x(u, \omega) \\
& =\varlimsup_{\substack{s \rightarrow t \\
s \in D}}\left(x(s, \omega)+\frac{1}{2} \alpha(s)\right) \geqq \varlimsup_{\substack{s \rightarrow t \\
s \in D}} x(s, \omega)+\frac{1}{2} a .
\end{aligned}
$$

But this is impossible, unless

$$
\varlimsup_{\substack{s \rightarrow t \\ s \in D}} x(s, \omega)=\infty
$$

that is, unless $\overline{\lim }_{s \rightarrow t} x(s, \omega)=\infty$.

Therefore

$$
P\left[\varlimsup_{s \rightarrow t} x(s, \omega)=\infty \text { for every } t \in D\right]=1 .
$$

Similarly we have

$$
P\left[\lim _{s \rightarrow t} x(s, \omega)=-\infty \text { for every } t \in D\right]=1 .
$$

This completes our proof, since $D$ is dense in $I$. 


\section{Proof of Theorem 4(a).}

Let $\alpha(t)$ be the oscillation function of a Gaussian process $x(t), 0<t<1$. Then we have

$$
P\left[W_{x}(t, \omega)=\alpha(t) \text { for every } t\right]=1
$$

by the definition of $\alpha(t)$ in Section 2. By the definition of $W_{x}(t, \omega)$, it holds that

so that we have

$$
\varlimsup_{s \rightarrow t} W_{x}(s, \omega) \leqq W_{x}(t, \omega),
$$

$$
\varlimsup_{s \rightarrow t} \alpha(s) \leqq \alpha(t),
$$

which shows that $\alpha$ is upper semi-continuous. Hence it follows that $T_{a}=\{t: \alpha(t) \geqq a\}$ is a closed subset of $[0,1]$. If $T_{a}-T_{\infty}$ contains a dense subset $D$ of an open interval for some $a>0$, then $D \subset T_{\infty}$ by Theorem 3, in contradiction with $D \subset T_{a}-T_{\infty}$. Therefore, $T_{a}-T_{\infty}$ is nowhere dense for $a>0$. This completes the proof of Theorem $4(a)$.

\section{Proof of Theorem $4(b)$.}

We shall denote the mean square norm of a random variable $x$ by $\|x\|$,

$$
\|x\|^{2}=E\left(x^{2}\right) .
$$

Consider a Brownian motion $B(t), 0 \leqq t \leqq 1$, and a stationary Gaussian process $S(t),-\infty<t<\infty$, with $E S(t)=0, E S(t)^{2}=1$ and $\alpha(t, S) \equiv \infty$. The existence of the latter process was proved by Belayev [1]. We can assume that these two processes are independent. Let $L$ be the \|\| -closure of all finite linear combinations of $B(t), 0 \leqq t \leqq 1$, and $S(t), 0 \leqq t \leqq 1$. It is clear that any process $x(t), 0 \leqq t \leqq 1$, such that $x(t) \in L$ for each $t$ is jointly Gauss distributed.

We shall prove Theorem 4(b) by constructing a Gaussian process $x(t) \in L, 0 \leqq t \leqq 1$, with $\alpha(t, x)=\alpha(t)$ for any given function

$$
\alpha:[0,1] \rightarrow[0, \infty] \text { satisfying }(\alpha, 1) \text { and }(\alpha, 2) \text {. }
$$

Let us start with some lemmas.

LeMma 6.1. Given $I=[u, v] \subset[0,1]$ and $\varepsilon>0$, we can construct a Gaussian process $x(t) \in L, 0 \leqq t \leqq 1$, satisfying

(a) $x(t, \omega)=0$ for $t \in[0,1]-I^{\circ}\left(I^{\circ}=\right.$ the interior of $\left.I\right)$,

(b) $\alpha(t, x)=\infty$ for $t \in I$,

(c) $\|x(t)\| \leqq \varepsilon$ for $t \in[0,1]$. 
Proof. Take a continuous function $f(t)$ such that $0<f(t)<\varepsilon$ in $I^{\circ}$ and $f(t)=0$ elsewhere. Then $x(t, \omega) \equiv f(t) S(t, \omega)$ is a Gaussian process satisfying our conditions.

Lemma 6.2. Given $0<a<\infty, \varepsilon>0$, and $I=[u, v] \subset[0,1]$, we can construct $a$ Gaussian process $x(t) \in L, 0 \leqq t \leqq 1$, satisfying the following conditions:

(a) $x(t, \omega)$ has continuous paths,

(b) $x(t, \omega)=0$ for $t \in[0,1]-I^{\circ}$,

(c) $E x(t)=0,\|x(t)\|<\varepsilon$ for every $t$,

(d) $P\left(\left|\sup _{I} x(t)-a\right|>\varepsilon\right)<\varepsilon$.

Such a process will be denoted by $x(t ; I, a, \varepsilon)$.

Proof. Let $B(t)$ be a Brownian motion and define a process $y(t)$ by

$$
\begin{aligned}
y(t) & =0, & & 0 \leqq t \leqq u, \\
& =\frac{a(B(t)-B(u))}{\left(2(t-u) \log \log (t-u)^{-1}\right)^{\frac{1}{2}}}, & & u \leqq t \leqq v^{\prime} \equiv \min (v, u+9), \\
& =\frac{a\left(B\left(v^{\prime}\right)-B(u)\right)}{\left(2\left(v^{\prime}-u\right) \log \log \left(v^{\prime}-u\right)^{-1}\right)^{\frac{1}{2}}}, & & v^{\prime} \leqq t \leqq 1 .
\end{aligned}
$$

Then $y(t)$ is jointly Gauss distributed with $E y(t)=0$ and the sample path of $y(t)$ is continuous except at $t=u$. The continuity in the mean follows from

$$
E\left[y(t)^{2}\right]=\frac{a^{2}}{2 \log \log (t-u)^{-1}} \rightarrow 0 \quad \text { as } t \downarrow u .
$$

By the law of the iterated logarithm we have

$$
P\left[\varlimsup_{t \downarrow u} y(t)=a\right]=1 .
$$

We now determine $u<s_{1}<s_{2}<s_{3}<s_{4}<v$ as follows. By taking $s_{4}$ sufficiently close to $u$, we have

$$
\begin{gathered}
E\left[y(t)^{2}\right]<\varepsilon^{2}, \\
P\left[\sup _{u<t<s_{4}} y(t)<a+\varepsilon\right]>1-\frac{1}{2} \varepsilon, \\
P\left[\sup _{u<t<s_{4}} y(t)>a-\varepsilon\right]>1-\frac{1}{2} \varepsilon .
\end{gathered}
$$


By taking $s_{2}$ sufficiently close to $u$ and $s_{3}$ sufficiently close to $s_{4}$, we have

$$
P\left[\sup _{s_{2} \leqq t \leqq s_{3}} y(t)>a-\varepsilon\right]>1-\frac{1}{2} \varepsilon .
$$

We shall take $s_{1}$ in $\left(u, s_{2}\right)$ arbitrarily.

Let $f(t)$ be a polygonal function of $t$ vanishing on $\left[0, s_{1}\right] \cup\left[s_{4}, 1\right]$, equal to 1 on $\left[s_{2}, s_{3}\right]$ and linear in each of $\left[s_{1}, s_{2}\right]$ and $\left[s_{3}, s_{4}\right]$. Then $x(t)=f(t) y(t)$ is a Gaussian process satisfying our conditions.

Lemma 6.3. Suppose that $\alpha_{1}(t)$ and $\alpha_{2}(t)$ satisfy $(\alpha, 1)$ and the following condition (stronger than $(\alpha, 2))$ :

$$
\left\{t: \alpha_{2}(t)>0\right\} \text { is nowhere dense. }
$$

For any Gaussian process $x_{1}(t)$ with $\alpha\left(t, x_{1}\right)=\alpha_{1}(t)$ and any $\varepsilon>0$, we can construct a Gaussian process $x_{2}(t) \in L, 0 \leqq t \leqq 1$, satisfying the conditions:

(a) $\alpha\left(t, x_{1}+x_{2}\right)=\alpha_{1}(t)+\alpha_{2}(t)$

(b) $\left\|x_{2}(t)\right\|<\varepsilon$,

(c) $P\left[\sup _{t}\left|x_{2}(t)\right|>\sup _{t} \alpha_{2}(t)\right]<\varepsilon$.

Proof. We can assume that $c=\sup \alpha_{2}(t)>0$. If otherwise, $x_{2}(t) \equiv 0$ will satisfy our conditions trivially.

Write $\alpha(t)$ for $\alpha_{1}(t)+\alpha_{2}(t)$. The set $\{(t, \alpha(t)): \alpha(t)>0\}$ is a subset of $[0,1] \times[0, \infty]$. Let $\left\{\left(t_{n}, \alpha\left(t_{n}\right)\right)\right\}_{n}$ be a countable dense subset of $\{(t, \alpha(t))$ : $\alpha(t)>0\}$. Then the sets $\left\{t_{n}\right\}_{n}$ are dense in $\{t: \alpha(t)>0\}$ and so dense in its closure $F$. By our assumptions $\left(\alpha, 2^{\prime}\right), F$ is nowhere dense and its complement $G$ is a dense open subset of $[0,1]$. Since $\alpha(t) \geqq \alpha\left(t, x_{1}\right)$, we have $\alpha\left(t, x_{1}\right)=0$ for $t \in G$, so that the sample path of $x_{1}(t)$ is continuous in $t \in G$ with probability one.

Using Theorem 2 and Lemma 4.1, we can find $\left\{t_{1 n}\right\}_{n}$ in $G$ tending to $t_{1}$ as $n \rightarrow \infty$ such that

$$
P\left[\varlimsup_{n \rightarrow \infty} x_{1}\left(t_{1 n}\right)=x\left(t_{1}\right)+\frac{1}{2} \alpha_{1}\left(t_{n}\right)\right]=1 .
$$

Since the path of $x_{1}(t)$ is continuous in $t \in G$, we can find, for each $t_{1 n}$, a closed interval $I_{1 n} \subset G$ containing $t_{1 n}$ in its interior such that

This implies

$$
P\left[x_{1}\left(t_{1 n}\right) \geqq \inf _{I_{1 n}} x_{1}(s) \geqq x_{1}\left(t_{1 n}\right)-2^{-n}\right]<2^{-n} .
$$

$$
P\left[\varlimsup_{n} \inf _{I_{1 n}} x_{1}(s)=\varlimsup_{n \rightarrow \infty} x_{1}\left(t_{1 n}\right)\right]=1
$$


by Borel-Cantelli's lemma. Thus we have

$$
P\left[\varlimsup_{n \rightarrow \infty} \inf _{I_{1 n}} x_{1}(s)=x\left(t_{1}\right)+\frac{1}{2} \alpha_{1}\left(t_{1}\right)\right]=1 .
$$

By taking $I_{1 n}$ sufficiently small, we can achieve that the $\left\{I_{1 n}\right\}_{n}$ are disjoint. Then $\Sigma_{n}\left|I_{1 n}\right| \leqq 1$ where $|\cdot|$ denotes length. Therefore $\left|I_{1 n}\right| \rightarrow 0$ and so $I_{1 n}$ tends to $t_{1}$ by $t_{1 n} \rightarrow t_{1}$.

It is clear that $\bigcup_{n} I_{1 n} \cup\left\{t_{1}\right\}$ is a closed set which does not contain $t_{2}$. Therefore we can find a neighborhood $U$ of $t_{2}$ which does not intersect this closed set. In the same way as above, we can find a sequence of disjoint intervals $I_{2 n} \subset G \cap U, n=1,2, \ldots$, tending to $t_{2}$ such that

$$
P\left[\varlimsup_{n \rightarrow \infty} \inf _{I_{2 n}} x_{1}(s)=x_{1}\left(t_{2}\right)+\frac{1}{2} \alpha_{1}\left(t_{2}\right)\right]=1 .
$$

Continuing this procedure we can get a double sequence of disjoint closed intervals $I_{k n} \subset G, k, n=1,2, \ldots$, such that $I_{k n}$ tends to $t_{k}$ as $n \rightarrow \infty$ for each $k$ and that

$$
P\left[\varlimsup_{n \uparrow \infty} \inf _{k n} x_{1}(s)=x_{1}\left(t_{k}\right)+\frac{1}{2} \alpha_{1}\left(t_{k}\right)\right]=1, \quad k=1,2, \ldots
$$

By removing a finite number of intervals from $\left\{I_{k n}\right\}_{n}$ we can achieve that $I_{k n} \subset\left(t_{k}-1 / k, t_{k}+1 / k\right)$ for each $k$.

Take $\varepsilon_{k n}>0$ such that

and set

$$
\sum_{k n} \varepsilon_{k n}<\min \left(\frac{1}{2} \varepsilon, \frac{1}{2} c\right)
$$

$$
x_{k n}(t)=x\left(t ; I_{k n}, a_{k n}, \varepsilon_{k n}\right) \quad(\text { see Lemma 6.2), }
$$

where $a_{k n}=\frac{1}{2} \alpha_{2}\left(t_{k n}\right)$ if this is finite and $=n$ otherwise, so that $a_{k n} \uparrow \frac{1}{2} \alpha_{2}\left(t_{k n}\right)$ as $n \rightarrow \infty$ for each $k$. We shall prove that

$$
x_{2}(t)=\sum_{k n} x_{k n}(t)
$$

is a Gaussian process satisfying our conditions.

Since this implies

$$
\begin{aligned}
x_{2}(t) & =x_{k n}(t) & & \text { on } I_{k n}, \quad k, n=1,2, \ldots, \\
& =0 & & \text { elsewhere }
\end{aligned}
$$

$x_{2}(t)$ is well-defined and $x_{2}(t) \in L$. Therefore $x_{2}(t)$ is jointly Gauss distributed, separable and measurable.

By $\left\|x_{k n}(t)\right\|<\varepsilon_{k n}$ and $\sum \varepsilon_{k n}<\infty$, the series $\sum_{k n} x_{k n}(t)$ converges in the 
mean uniformly in $t$ and so $x_{2}(t)$ is continuous in the mean and we have

which proves (b).

$$
\left\|x_{2}(t)\right\|<\sum_{k n}\left\|x_{k n}(t)\right\|<\varepsilon
$$

Observing that

$$
\begin{aligned}
P\left[\sup _{t} x_{2}(t)>c\right] & \leqq \sum_{k n} P\left[\sup _{t \in I_{k n}} x_{2}(t)>c\right] \\
& \leqq \sum_{k n} P\left(\sup _{t} x_{k n}(t)>a_{k n}+\varepsilon_{k n}\right) \\
& \leqq \sum_{k n} \varepsilon_{k n}<\frac{1}{2} \varepsilon,
\end{aligned}
$$

we have

$$
P\left(\sup _{t}\left|x_{2}(t)\right|>c\right)<\varepsilon,
$$

since the probability law of the sample path of $x_{2}(t)$ is symmetric by $E x_{2}(t)=0$. Thus (c) is proved.

Now we shall prove that $\alpha\left(t, x_{2}\right) \leqq \alpha_{2}(t)$.

If $t_{0} \in G$, then $t_{0}$ has a positive distance from $F$. Since $I_{k n}$ is in the 1/k-neighborhood of $t_{k} \in F$, a small neighborhood $U(\subset G)$ of $t_{0}$ does not intersect $I_{k n}$ with $k \geqq k_{0}, n=1,2, \ldots$, for some $k_{0}$. Since $I_{k n} \rightarrow t_{k} \in F$ for each $k, U$ can intersect only a finite number of intervals among $\left\{I_{k n}\right\}_{k n}$. Let us denote these intervals by $I_{k(i), n(i)}, i=1,2, \ldots, m$. Then $x_{2}(t)$ is the sum of $x_{k(i), n(i)}, i=1,2, \ldots, m$, as far as $t$ lies in $U$. Therefore the path of $x_{2}(t)$ is continuous in $U$ and so $\alpha\left(t_{0}, x\right)=0 \leqq \alpha_{2}\left(t_{0}\right)$.

If $t_{0} \in F$, then $x_{2}\left(t_{0}\right)=0$ by our construction. Take an arbitrary $\delta>0$. Then there exists a neighborhood $U_{1}$ of $t_{0}$ such that

$$
\sup _{s \in U_{1}} \alpha_{2}(s)<\alpha_{2}\left(t_{0}\right)+\delta .
$$

Take a neighborhood $U_{2}$ of $t_{0}$ such that $\bar{U}_{2} \subset U_{1}$. Then the distance $\varrho\left(U_{2}, U_{1}^{c}\right)$ is positive. Since the $\left\{I_{k n}\right\}_{k n}$ are disjoint and $\Sigma_{k n}\left|I_{k n}\right| \leqq 1$, we have only a finite number of intervals among $\left\{I_{k n}\right\}_{k n}$ with the length $\geqq \varrho\left(U_{2}, U_{1}^{c}\right)$ and only such intervals can intersect both $U_{2}$ and $U_{1}{ }^{c}$. Since each $I_{k n}$ has positive distance from $t_{0}$, we have a neighborhood $U_{3}\left(\subset U_{2}\right)$ of $t_{0}$ such that $I_{k n} \subset U_{1}$ as far as $I_{k n}$ intersects $U_{3}$.

Write $\Sigma^{\prime}$ for the summation over those indices $(k, n)$ for which $I_{k n}$ intersects $U_{3}$. Then any interval $I_{k n}$ with the index $(k, n)$ appearing in $\Sigma^{\prime}$ is contained in $U_{1}$. By taking $U_{3}$ small enough, we can achieve that

$$
\sum_{k n}^{\prime} \varepsilon_{k n}<\delta
$$


We then have

$$
\begin{aligned}
P\left[\sup _{s \in U_{3}} x_{2}(s)\right. & \left.>\frac{1}{2} \alpha_{2}\left(t_{0}\right)+2 \delta\right] \\
& \leqq \Sigma^{\prime} P\left[\sup x_{k n}(s)>\sup _{s \in U_{1}} \alpha_{2}(s)+\delta\right] \\
& \leqq \Sigma^{\prime} P\left(\sup x_{k n}(s)>a_{k n}+\varepsilon_{k n}\right)<\sum^{\prime} \varepsilon_{k n}<\delta,
\end{aligned}
$$

by the construction in Lemma 6.2. Therefore we get

$$
P\left[\varlimsup_{s \rightarrow t_{0}} x_{2}(s)>\frac{1}{2} \alpha_{2}\left(t_{0}\right)+2 \delta\right]<\delta
$$

for every $\delta>0$. Letting $\delta \downarrow 0$, we have

$$
P\left[\varlimsup_{s \rightarrow t_{0}} x_{2}(s) \geqq x_{2}\left(t_{0}\right)+\frac{1}{2} \alpha_{2}\left(t_{0}\right)\right]=0 . \quad\left(\text { Note } x_{2}\left(t_{0}\right)=0 .\right)
$$

This implies that $\alpha\left(t_{0}, x_{2}\right) \leqq \alpha_{2}\left(t_{0}\right)$ by Theorem 2 .

Thus $\alpha\left(t, x_{2}\right) \leqq \alpha_{2}(t)$ is proved for every $t$. By the definition of the oscillation function we have

$$
\alpha\left(t, x_{1}+x_{2}\right) \leqq \alpha\left(t, x_{1}\right)+\alpha\left(t, x_{2}\right) \leqq \alpha_{1}(t)+\alpha_{2}(t)=\alpha(t) .
$$

We shall now prove that

$$
\alpha\left(t, x_{1}+x_{2}\right) \geqq \alpha(t) .
$$

Consider first the case $t=t_{k}$. It holds that

$$
\begin{aligned}
P\left[\varlimsup_{s \rightarrow t_{k}}\right. & \left.\left(x_{1}(s)+x_{2}(s)\right) \geqq x_{1}\left(t_{k}\right)+x_{2}\left(t_{k}\right)+\frac{1}{2} \alpha\left(t_{k}\right)\right] \\
& \geqq P\left[\varlimsup_{n \rightarrow \infty} \sup _{I_{k n}}\left[x_{1}(s)+x_{2}(s)\right] \geqq x_{1}\left(t_{k}\right)+x_{2}\left(t_{k}\right)+\frac{1}{2} \alpha\left(t_{k}\right)\right] \\
& \geqq P\left[\varlimsup_{n \rightarrow \infty}\left(\inf _{I_{k n}} x_{1}(s)+\sup _{I_{k n}} x_{2}(s)\right) \geqq x_{1}\left(t_{k}\right)+x_{2}\left(t_{k}\right)+\frac{1}{2} \alpha\left(t_{k}\right)\right] \\
& \geqq P\left[\varlimsup_{n \rightarrow \infty} \inf _{I_{k n}} x_{1}(s)+\underset{\lim _{n \rightarrow \infty}}{\left.\sup _{I_{k n}} x_{2}(s) \geqq x_{1}\left(t_{k}\right)+x_{2}\left(t_{k}\right)+\frac{1}{2} \alpha\left(t_{k}\right)\right] .}\right.
\end{aligned}
$$

Since we have

$$
P\left[\sup _{I_{k n}} x_{2}(s)<a_{k n}-\varepsilon_{k n}\right]<\varepsilon_{k n}
$$

by virtue of $x_{2}(s)=x_{k n}(s)$ on $I_{k n}$, we get

$$
P\left[\lim _{n \rightarrow \infty} \sup _{I_{k n}} x_{2}(s) \geqq x_{2}\left(t_{k}\right)+\frac{1}{2} \alpha_{2}\left(t_{k}\right)\right]=1
$$


by $x_{2}\left(t_{k}\right)=0$ and $\Sigma_{n} \varepsilon_{k n}<\infty$. By (6.1), (6.2) and (6.3) we get

$$
P\left[\varlimsup_{s \rightarrow t_{k}}\left(x_{1}(s)+x_{2}(s)\right) \geqq x_{1}\left(t_{k}\right)+x_{2}\left(t_{k}\right)+\frac{1}{2}\left(t_{k}\right)\right]=1,
$$

that is, by Theorem 2 ,

$$
\alpha\left(t_{k}, x_{1}+x_{2}\right) \geqq \alpha\left(t_{k}\right) .
$$

For $t \neq t_{k}, k=1,2, \ldots$, the point $(t, \alpha(t))$ is an accumulation point of $\left(t_{n}, \alpha\left(t_{n}\right)\right), n=1,2, \ldots$. Therefore we have a subsequence $\left\{s_{n}\right\}$ of $\left\{t_{n}\right\}$ such that

$$
\alpha(t)=\lim _{n} \alpha\left(s_{n}\right) .
$$

By Theorem 4 (a) $(\alpha, 1)$ we have

$$
\alpha\left(t, x_{1}+x_{2}\right) \geqq \varlimsup_{n} \alpha\left(s_{n}, x_{1}+x_{2}\right)=\varlimsup_{n} \alpha\left(s_{n}\right)=\alpha(t),
$$

which completes the proof of Lemma 6.3.

Now we shall come back to the proof of Theorem 4 (b). We shall first assume

$\left(\alpha, 2^{\prime \prime}\right) \quad\{t: \alpha(t) \geqq c\}$ is nowhere dense for every $c>0 ;$

this is stronger than $(\alpha, 2)$ but weaker than $\left(\alpha, 2^{\prime}\right)$. Set

$$
\begin{aligned}
& \alpha_{0}(t)=0 \\
& \alpha_{1}(t)=\max \left(\alpha(t), \frac{1}{2}\right)-\frac{1}{2}, \\
& \alpha_{n}(t)=\max \left(\min \left(\alpha(t), 2^{-n+1}\right), 2^{-n}\right)-2^{-n}, \quad n=2,3, \ldots .
\end{aligned}
$$

It is then easy to verify the following properties of $\alpha_{n}(t)$ :

$$
\begin{aligned}
& 0 \leqq \alpha_{n}(t) \leqq 2^{-n-1}, \quad n=2,3, \ldots, \\
& \sum_{0}^{n} \alpha_{i}(t) \uparrow \alpha(t) \\
& \left\{t: \alpha_{n}(t)>0\right\} \text { is nowhere dense } .
\end{aligned}
$$

Starting with the Gaussian process $x_{0}(t) \equiv 0$ whose oscillation function is $\alpha_{0}(t) \equiv 0$, we can use Lemma 6.3 to define a sequence of Gaussian processes $x_{n}(t) \in L, 0 \leqq t \leqq 1, n=1,2, \ldots$, satisfying

(a) $\alpha\left(t, \sum_{0}^{n} x_{i}\right)=\sum_{0}^{n} \alpha_{i}(t)$,

(b) $\left\|x_{n}(t)\right\|<2^{-n}$,

(c) $P\left[\sup _{t}\left|x_{n}(t)\right|>\sup _{t} \alpha_{n}(t)\right]<2^{-n}$.

Now set

$$
x(t)=\Sigma_{n} x_{n}(t) .
$$

By Borel-Cantelli's lemma it follows from (c) and $\alpha_{n}(t) \leqq 2^{-n-1}, n \geqq 2$, that this infinite series converges uniformly in $t$ with probability one. 
Therefore $x(t)$ is well-defined, separable, measurable and jointly Gauss distributed, and we have

$$
\alpha(t, x)=\lim _{n} \alpha\left(t, \Sigma_{0}^{n} x_{i}\right)=\lim _{n} \sum_{0}^{n} \alpha_{i}(t)=\alpha(t) .
$$

It follows from (b) that this infinite series converges also in the mean, uniformly in $t$, so that $x(t)$ is continuous in the mean.

We shall now remove the assumption that $T_{c}=\{t: \alpha(t) \geqq c\}$ is nowhere dense.

Let $I_{1}, I_{2}, \ldots$ be the maximal intervals contained in the set $T_{\infty}=$ $\{t: \alpha(t)=\infty\}$. Since, by $(\alpha, 2),\{t: c \leqq \alpha(t)<\infty\}$ is nowhere dense, $I_{1}, I_{2}, \ldots$ are also the maximal intervals contained in the set $T_{c}(c>0)$.

We now define

$$
\begin{aligned}
\beta(t) & =0, & & t \in I_{n}^{\circ}, \quad n=1,2, \ldots, \\
& =\alpha(t) & & \text { elsewhere } .
\end{aligned}
$$

Then $\beta(t)$ satisfies $(\alpha, 1)$ and $\left(\alpha, 2^{\prime \prime}\right)$. Therefore we can construct a Gaussian process $y(t) \in L, 0 \leqq t \leqq 1$, such that $\alpha(t, y)=\beta(t)$, as we proved above.

By Lemma 6.1, we can construct a sequence of Gaussian processes $y_{n}(t) \in L, 0 \leqq t \leqq 1, n=1,2, \ldots$, such that $\alpha\left(t, y_{n}\right)=\infty$ on $I_{n}, y_{n}(t)=0$ on $[0,1]-I_{n}^{\circ}$ and $\left\|y_{n}(t)\right\|<2^{-n}$. Now consider

Then

$$
x(t)=y(t)+\Sigma_{n} y_{n}(t) .
$$

$$
\begin{aligned}
x(t) & =y_{n}(t)+y(t), & & t \in I_{n}^{\circ}, \quad n=1,2, \ldots, \\
& =y(t), & & \text { elsewhere, }
\end{aligned}
$$

and $x(t) \in L, 0 \leqq t \leqq 1$. Therefore $x(t)$ is jointly Gaussian, measurable and separable. Its continuity in the mean follows from $\left\|y_{n}(t)\right\|<2^{-n}$.

To complete our proof, we need only to show that $\alpha(t, x)=\alpha(t)$. Since $x(t)=y(t)$ on the set $G=[0,1]-\bar{U}_{n} I_{n}$ which is open in $[0,1]$, we have

$$
\alpha(t, x)=\alpha(t, y)=\beta(t)=\alpha(t), \quad t \in G .
$$

Since $x(t)=y_{n}(t)+y(t)$ and $y(t)$ is continuous in $I_{n}^{\circ}$, we have

$$
\alpha(t, x)=\alpha\left(t, y_{n}\right)=\infty=\alpha(t), \quad t \in \bigcup_{n} I_{n}^{\circ} .
$$

If $t \in U_{n} I_{n}-U_{n} I_{n}^{\circ}$, then $t$ is an accumulation point of $\bigcup_{n} I_{n}^{\circ}$ and so we get

by Theorem 4 (a) $(\alpha, 1)$.

$$
\alpha(t, x) \geqq \varlimsup_{\substack{s \rightarrow t \\ s \in U I_{n^{\circ}}}} \alpha(s, x)=\infty
$$




\title{
REFERENCE
}

1. Yu. K. Belayev, Continuity and Hölder's conditions for sample functions of stationary Gaussian processes, Proc. 4th Berkeley Sympos. Math. Statist. and Prob., Vol. 2., 23-33. Univ. Calif. Press, 1961.

\author{
AARHUS UNIVERSITY, DENMARK \\ $\triangle N D$ \\ KOBE UNIVERSTTY, JAPAN
}

